\title{
Ringen um das Existenzminimum
}

Die konträren politischen Reaktionen auf das Urteil des Bundesverfassungsgerichts vom 9. Februar 2010, wonach die bisherige Ermittlung der Kinder- und Erwachsenenregelsätze mit dem Grundrecht auf ein menschenwürdiges Existenzminimum nicht vereinbar ist, haben es deutlich gemacht: Die Politik und letztlich die Bevölkerung ist tief gespalten in der Frage, wie viel Geld man in Deutschland zu einem menschenwürdigen Leben und einem Mindestmaß an soziokultureller Teilhabe benötigt. Allein die Vorstellungen über die Höhe eines Regelsatzes für Alleinstehende variieren im Moment zwischen $132 €$ und $500 €$ pro Monat. Ebenfalls umstritten ist, wie viel Geld Familien - insbesondere Kinder - benötigen, außerdem die Höhe der notwendigen Unterkunftskosten, und ob es überhaupt nur bei gleichzeitiger Ableistung eines öffentlichen Arbeitsdienstes gezahlt werden soll. Warum diese Uneinigkeit fast 50 Jahre nach Einführung des Sozialhilfeanspruchs, mit dem parallel die systematische und mehr oder weniger transparente Ermittlung der verschiedenen Regel- und Sonderbedarfe vorangetrieben wurde?

Die Erinnerung an den Verfassungsauftrag veranlasst darüber hinaus einige zur umgehenden Warnung vor spätrömischer Dekadenz durch leistungsloses Einkommen. Als Erstes wird die Gutscheinausgabe an Hartz-IV-Eltern gefordert, bevor auch nur der fehlende Bildungsbedarf ermittelt ist. Schon verbreiten Politiker wie Wissenschaftler die Befürchtung, dass eine solide staatliche Existenzsicherung nur ungebildete Unterschichten zur Vermehrung anregt und die Einwanderung von „Niedrigstleistern des Auslands“ bewirkt. „Mehr Geld vermehrt Armut", werden wir belehrt, was zu dem messerscharfen Umkehrschluss führt, dass weniger Geld zu weniger Armut führe, wozu ausgerechnet die insoweit blühenden Lebensverhältnisse in den USA herangezogen werden. Starke negative Arbeitsanreize seien zu erwarten, wenn der Regelsatz auch nur von derzeit $359 €$ auf $420 €$ angehoben würde, obwohl sich der größere Teil der auf $10 \mathrm{Mrd}$. geschätzten Mehrausgaben aus den dadurch bewirkten Steuermindereinnahmen und den aufstockenden Leistungen für Niedrigverdiener zusammensetzen würde. Das würde zwar wirksam Armut lindern und die Wirtschaft könnte über die Löhne an der Finanzierung beteiligt werden, aber eine noch so sachgerechte Begründung der notwendigen Bedarfe interessiert diejenigen nicht, die den Regelsatz absenken wollen. In der Auseinandersetzung fehlt etwas: ein sozialer Grundkonsens, ein gemeinsames Menschenbild, - eben die Orientierung an der Verfassung. Der soziale Rechtsstaat und die Menschenwürde werden weiter beschworen, aber die Umsetzung in Euro und Cent wird abgelehnt.

Die Bestimmung des Existenzminimums in Deutschland hatte immer eine Besonderheit: Sie wurde getrennt von der Arbeitswelt und ihren Akteuren, getrennt von der Lohnfrage verhandelt.
Sozialhilfe war eine staatliche Aufgabe, für die sich Verwaltung, Wohlfahrtsverbände und Sozialpolitik interessierten, nicht aber die Wirtschaftsakteure.

Natürlich wäre es besser, ein ganzheitlicheres Konzept zu verfolgen, wie es F. D. Roosevelt mit seiner Second Bill of Rights postulierte: das Recht auf ausreichende Bezahlung von Arbeit und auskömmliche Erträge für kleine Selbstständige verbunden mit dem Recht auf Sicherung in allen Gebieten der Sozialversicherung und dem Recht auf gute Bildung. Doch das ist bisher nicht umgesetzt. Eine Übersicht aus Luxemburg zeigt, wie bezogen auf einen Lebenshaltungskostenindex differenzierte Mindestlöhne, Renten, Familienleistungen und Mindesteinkommen für Nichterwerbstätige ein abgestimmtes Ganzes bilden können. Und niemand kommt auf die Idee, das eine gegen das andere auszuspielen. Dieser Zusammenhang wird in Deutschland bisher nicht gesehen. Aber gerade wegen eines fehlenden Existenz sichernden Mindestlohns wirkt sich die Ermittlung des Existenzminimums nicht nur auf Unterschichten und Randexistenzen aus, sondern als Referenzgröße genauso auf die Arbeitenden. Vorerst ist das Niveau der Sozialhilfe die einzige Größe, die nicht nur den Steuerfreibetrag bestimmt, sondern den Basisschutz mitübernehmen und Einkommen in vielen Fällen aufstocken muss.

Die Auseinandersetzung um die Bestimmung und Höhe dieses Existenzminimums geht nun in die nächste Runde. Die bisher herangezogene Einkommens- und Verbrauchsstatistik (EVS) ist nicht nur ungeeignet zur Bestimmung des Existenzminimums von Kindern, sie benötigt auch ergänzende Untersuchungen. Es werden jetzt schon zu viele herangezogen, die selbst im Erhebungszeitraum unter der Sozialhilfeschwelle lebten, zu viele, die alt und nicht mehr erwerbsfähig sind. Eine Anpassung an die Lebenshaltungskosten ist seit Jahren unterblieben.

Es geht dabei zunächst nur um die Bedarfe der Nichterwerbstätigen. Aber dabei darf man nicht mehr stehen bleiben, denn es geht auch um die Mindestbedarfe der Erwerbstätigen - Arbeitnehmer und Selbstständiger. Diese sind höher und müssen genauso sorgfältig ermittelt und geschützt werden. Dann erst wird diese Spaltung zwischen Niedrigeinkommensbeziehern und Erwerbslosen beendet, die durch viele sozialpolitische Versäumnisse und Eingriffe vertieft worden ist. Die Uneinigkeit über das Existenzminimum ist Ausdruck des fehlenden Grundkonsenses über das Menschenbild unserer Verfassung und des fehlenden ganzheitlichen Konzepts zur Armutsvermeidung.

Helga Spindler, Prof. Dr. jur., lehrt am Institut für soziale Arbeit und Sozialpolitik der Universität Duisburg-Essen. Arbeitsschwerpunkte: Öffentliches Recht, Sozialrecht, Arbeitsrecht. e-mail: helga.spindler@uni-due.de 